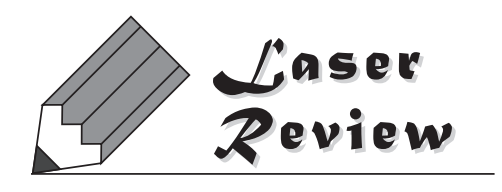

\title{
High-Performance Solid-State and Fiber Lasers Controlled by Volume Bragg Gratings
}

\author{
Leonid GLEBOV \\ CREOL/ the College of Optics and Photonics, University of Central Florida, \\ 4000 Central Florida Blvd. Orlando, FL, 32816-2700 USA
}

(Received July 1, 2013)

\begin{abstract}
The paper is a survey of properties and applications of optical elements which are volume Bragg gratings (VBGs) recorded in photo-thermo-refractive (PTR) glass. These elements are narrow band filters that operate in spectral, angular and temporal domains. They provide spectral narrowing, stabilization and tuning of laser radiation; narrowing of divergence and beam steering; stretching, compression and temporal shaping of laser pulses, integration of different laser components in the same material and fabrication of monolithic solid state lasers; and passive and active coherent combining along with high density spectral combining of fiber lasers.
\end{abstract}

Key Words: Volume Bragg gratings, PTR glass, Fiber and solid state lasers

\section{Introduction}

Exponential growth of industrial and medical applications of solid state and fiber lasers results in a strong demand for increasing power and brightness of laser sources concurrently with strong requirements for compactness and robustness. A number of methods are developed and successfully used for spectral and spatial filtering in laser resonators, pulse shaping and beam combining. However, conventional methods of laser control are often resulted in increasing complexity and decreasing robustness of laser systems.

Volume Bragg gratings (VBGs) are new optical components that appeared on laser market several years ago and already have found wide applications enabling record parameters in different areas of laser design. The goal of this survey is to show the current stage of the VBG technology and its laser applications. Therefore, this paper does not include the whole list of relevant publications and the references in this survey are used to provide necessary technical details but not to demonstrate priority of publications.

\section{Volume Bragg gratings in photo-thermo-refractive glass}

While basic optical properties and benefits of thick VBGs were understood at very early stages of laser development, ${ }^{1)}$ real applications were limited by the absence of a proper photosensitive material that should provide necessary refractive index increment, low losses, and high tolerance to laser radiation and harsh environment. The situation was changed when a photo-thermo-refractive (PTR) glass with high optical homogeneity and low losses was created, ${ }^{2)}$ high efficiency VBGs were recorded, ${ }^{3)}$ and their applications for lasers were demonstrated. ${ }^{4)}$ Intensive study and wide applications of VBGs recorded in PTR glass resulted in multiple original publications and several surveys published at different stages of the VBG technology development. ${ }^{5-7)}$

A uniform VBG is a trivial phase volume hologram produced by recording of an interference pattern of two collimated beams. This recording results in creation of numerous planar layers with modified refractive index in the volume of a photosensitive optical material. These layers provide resonant diffraction of optical beams that is described in ${ }^{1)}$ and detailed for engineering calculations in. ${ }^{89}$ Diffraction of radiation inside of this grating occurs if Bragg conditions are satisfied:

$$
\sin \theta_{m}=\frac{\lambda}{2 n d}
$$

where $\theta_{m}$ is an angle inside of the photosensitive medium between a plane of constant refractive index and a direction of beam propagation, $\lambda$ is wavelength, $n$ is refractive index and $d$ is a grating period. It is important to note that properties of volume diffraction gratings are dramatically different from those of conventional surface diffraction gratings. Therefore, applications of VBGs are dramatically different from those of surface diffraction gratings. Let us consider the main types of VBGs and their basic properties.

A transmitting VBG (Fig. 1) recorded in a plane-parallel glass plate is a volume diffractive element where both transmitted and diffracted beams cross the back surface. Diffraction occurs only if both a wavelength $\left(\lambda_{2}\right)$ and an incident angle $\left(\theta_{2}\right)$ of the launched beam correspond to Bragg condition (Eq. 1). Contrary to surface gratings, at all other combinations of incident angles and wavelengths beams transmit the VBG without changing the direction of propagation. For the same VBG, an incident angle for a particular wavelength could be varied from normal to grazing because the VBG can be arbitrary oriented inside of a glass plate (by changing of recording angles or by different cutting a recorded grating from an exposed glass slab). Grating orientation can be changed in 


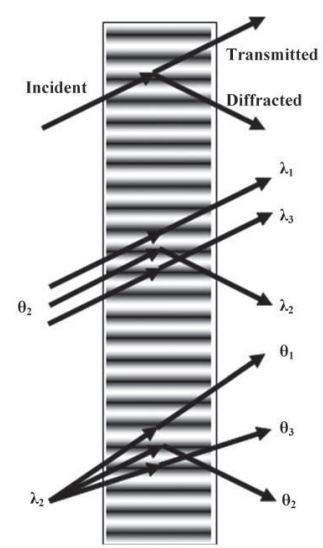

Fig. 1 Transmitting volume Bragg grating (VBG). Diffracted beam crosses the back surface. An incident beam with wavelength $\lambda_{2}$ approaching a VBG at incident angle $\theta_{2}$ is diffracted. All other beams with different wavelengths and/or different incident angles are transmitted through the VBG without changing direction of propagation.

such manner that diffracted beam would cross the front surface of the VBG (Fig. 2). This grating is a reflecting VBG or a Bragg mirror. Contrary to surface gratings where diffraction occurs for all wavelengths and angular dispersion can be about $1 \mathrm{deg} / \mathrm{nm}$, VBGs deflect a narrow line for large angles up to $180^{\circ}$ (Fig. 1, middle, for $\theta_{2}$ approaching $90^{\circ}$ ). This means that angular separation of adjacent narrow lines could be orders of magnitude higher if compared to that for surface gratings. For example, a Bragg mirror with spectral selectivity of $0.1 \mathrm{~nm}$ deflecting beam for $180^{\circ}$ provides angular dispersion of $1800 \mathrm{deg} / \mathrm{nm}$. Increasing grating thickness, decreasing grating period or decreasing angle of propagation $\left(\theta_{m}\right.$ in Eq. 1) result in narrowing the spectral selectivity of VBGs. ${ }^{1,89}$ For VBGs recorded in PTR glass, where thickness could be varied from fractions of millimeter to several centimeters, spectral selectivity could be varied from tens of nanometers for thin transmitting gratings with large period and grazing angle of propagation to tens of picometers for thick reflecting gratings with small period and normal angle of propagation.

An important feature of VBGs is their ability to provide not only spectral but also angular selectivity (Fig. 1, bottom). Conventional angular filtering is produced by spatial filters that include a lens for Fourier transform from angles to spatial coordinates, spatial filtering, and Fourier transform back to an original beam. A VBG works in angular space (or in space of wave vectors) and it provides direct angular filtering. Transmitting VBGs are slits in angular space. Their angular selectivity could be narrowed down to tens microradians. Reflect-

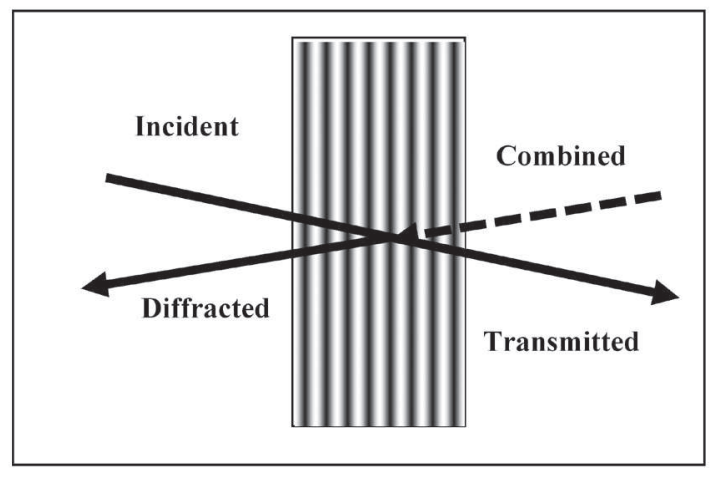

Fig. 2 Reflecting VBG. Diffracted beam crosses the front surface. ing VBGs are round diaphragms in angular space and their angular selectivity could be narrowed down to few milliradians.

Dependences of diffraction efficiency on wavelengths and incident angles for VBGs are not smooth functions as they are for surface gratings. An example of a diffraction efficiency spectrum for a reflecting VBG is shown in Fig. 3. It has a sharp central maximum and several side lobes separated by points with zero efficiency. ${ }^{1,8,9)}$ An angular response of this VBG has the same shape. Similar spectra are observed for transmitting VBGs. This is a key feature that enables the use of VBGs for spectral beam combining and for angular magnification in laser beam steerers.

PTR glass enables multiplication of high efficiency VBGs in the same volume. Two gratings recorded in a glass plate with the same direction of grating vectors but with different periods produce a moiré pattern (Fig. 4, top) where spatial profile of refractive index has a fast-frequency component with a period average of periods of recording interference patterns and slow-frequency envelope determined by difference of those periods (Fig. 4, bottom). A fraction of moiré pattern between two zeros of a slow-frequency envelope is a Bragg mirror with apodized refractive index modulation profile. ${ }^{10)}$ Two such fractions correspond to a Fabry-Perot interferometer. Unique features of such an interferometer are automatic alignment of "mirrors" naturally having a phase shift of $\pi$ and a single transmission maximum. The narrowest band of such a filter having thickness of $6 \mathrm{~mm}$ was $9 \mathrm{pm}$ at $633 \mathrm{~nm}$.

It is possible in the same glass plate to record two or more gratings with different Bragg wavelengths that are oriented to

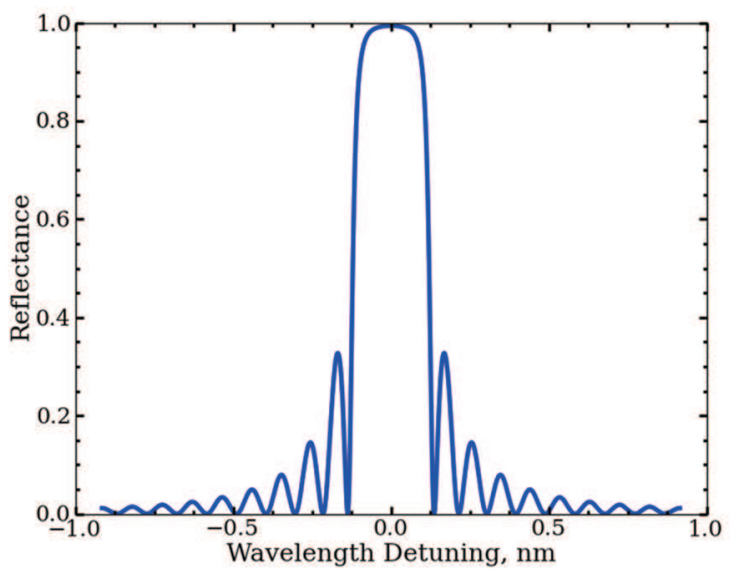

Fig. 3 The spectrum of diffraction efficiency for a reflecting VBG with a period of $360 \mathrm{~nm}$, thickness of 4.0 $\mathrm{mm}$ and refractive index modulation of $270 \mathrm{ppm}$ for $\lambda=1064 \mathrm{~nm}$ and $\theta=10^{\circ}$.
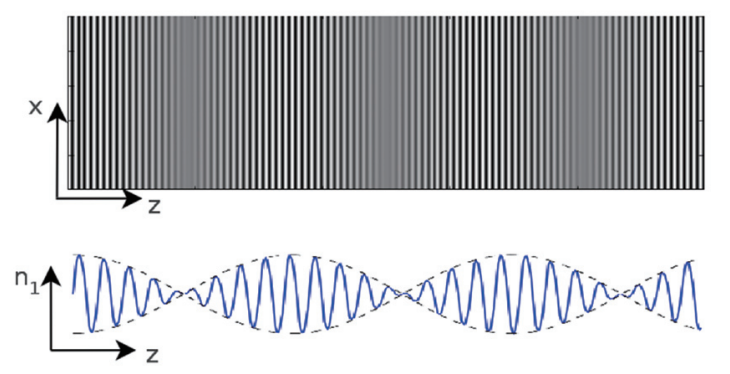

Fig. 4 Moiré grating recorded by sequential exposure to UV interference patterns with different periods (top) and spatial profile of refractive index (bottom, not in scale). 
have a common Bragg angle (Fig. 5, top). ${ }^{11,12}$ While these VBGs are overlapped in the same space, they are optically independent. Therefore, illuminating this complex element along a normal to the front surface at fixed wavelengths would result in diffraction of the beams with proper wavelengths in different directions. Figure 5, bottom demonstrates that a number of such VBGs with high efficiency can be recorded in PTR glass. It is clear that illuminating this grating by beams with proper wavelengths launched at proper angles would result in combing of those beams within the same aperture along the arrow in Fig. 5, top. This is one of the promising approaches for spectral beam combining (multiplexing).

It is possible in the same glass plate to record several gratings with the same Bragg wavelengths that are oriented to have a common Bragg angle. ${ }^{13,14)}$ These gratings are optically dependent because diffraction to the common Bragg angle would cause further diffraction to another grating. Figure 6 shows two identical gratings that work as a 1:2 beam splitter if
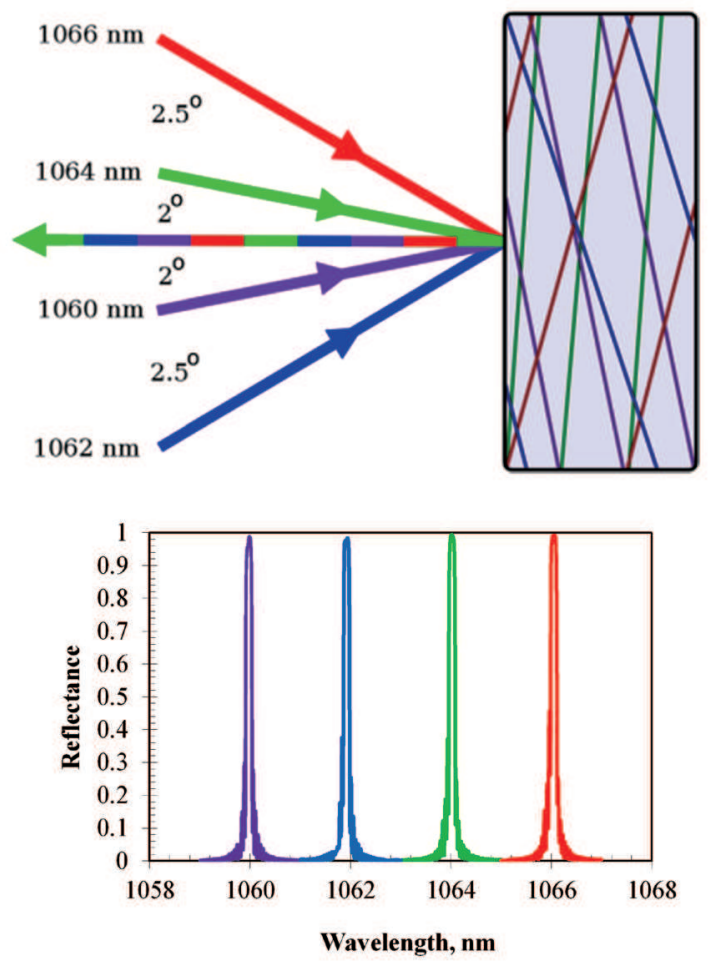

Fig. 5 Multiplexed reflecting VBG that includes four gratings with different periods and a common Bragg angle (top) and its reflection spectrum if illuminated along the normal to the front surface (bottom).

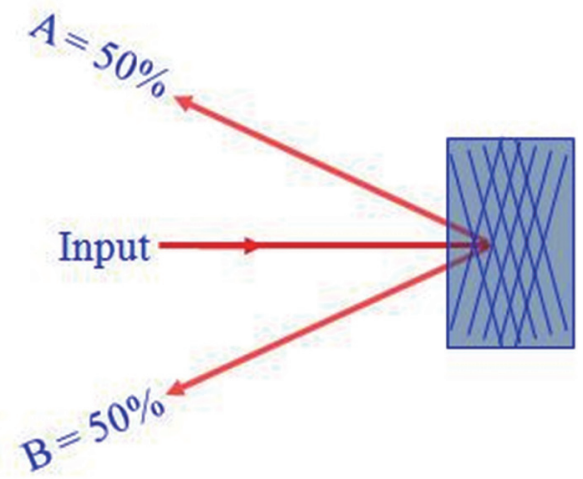

Fig. 6 Two identical VBGs recorded symmetrically in PTR glass act like a 1:2 splitter/combiner when the Bragg conditions are satisfied.

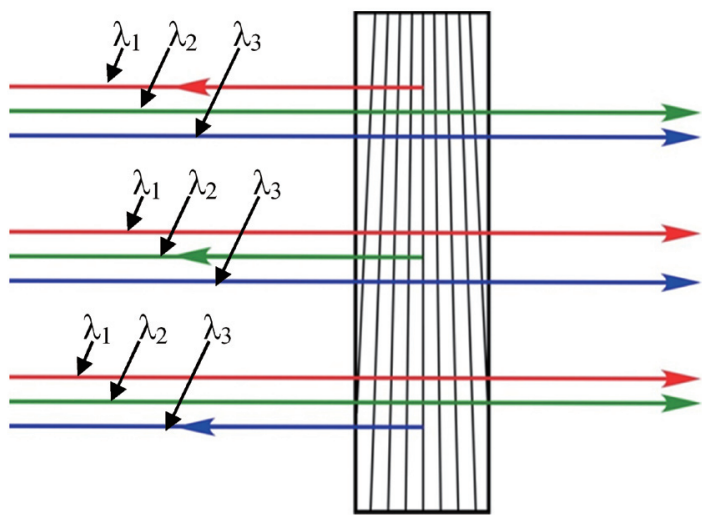

Fig. 7 Transverse chirped VBG with lateral variation of Bragg wavelength.

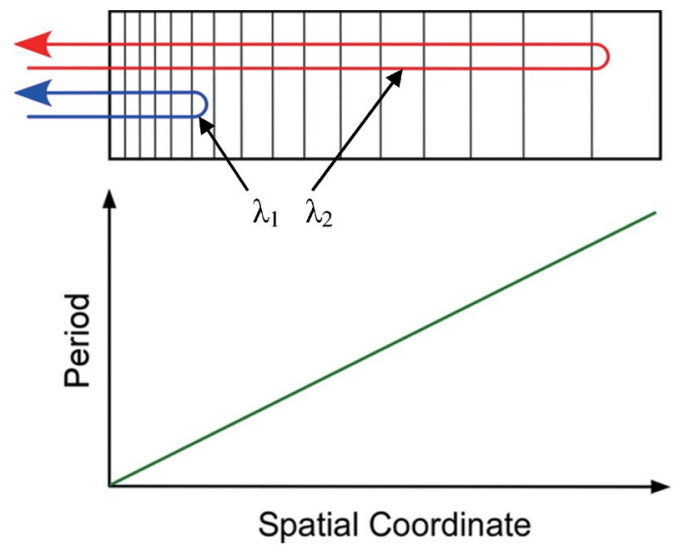

Fig. 8 Longitudinal chirped VBG (top) with period linearly varied in direction of beam propagation (bottom).

illuminated the arrow from left to right. However, illumination of this multiplexed grating by coherent beams launched from directions $\mathrm{A}$ and $\mathrm{B}$ would result in coherent combining within a common aperture. Multiplexed gratings that provide splitting 1:2, 1:4 and 1:5 were recorded. Such elements enable coherent combining.

While uniform VBGs are recorded using an interference pattern produced by two collimated beams, it is possible to combine a divergent beam with and a convergent one. In this case the interference pattern consists of dark and bright planes with a period gradually changed in direction perpendicular to a bisector of the recording beams. If convergence and divergence angles are equal, the resulting VBG would have a grating with a linearly varied period. If the period variation directed across the aperture, it is a transverse chirped reflecting VBG or a Bragg mirror with a variable resonant wavelength (Fig. 7). Such a grating being placed as an output coupler for a laser array would lock it to a series of predetermined wavelengths. This output coupler being slid in lateral direction would tune emission wavelengths. If period variation is directed along the beam propagation, it is a longitudinal chirped reflecting VBG (Fig. 8). Such a grating can work as a wide band filter with spectral width extended up to tens of nanometers or as a pulse stretcher/compressor.

\section{Spectral narrowing, stabilization and tuning of laser emission}

The use of reflecting Bragg gratings as narrow band output 
couplers in external resonators for different types of lasers is widely studied and published. One of the important applications is the use of spectral narrowing and stabilization for semiconductor diodes, bars and stacks to improve stability and efficiency of pumping solid state and fiber lasers. ${ }^{15,16,5)}$ It was found that PTR output couplers with thickness from parts to few millimeters provide efficient spectral locking of laser diodes at the level of from 60 to $100 \mathrm{pm}$. Emission wavelength does not depend on temperature of a diode. Efficiency of a volume Bragg laser diode usually exceeds $95 \%$ of efficiency of an original diode at power level of several watts. Locking of diode bars and stacks with total power up to $0.5 \mathrm{~kW}$ were demonstrated with efficiency above $90 \%$. The narrowest high power laser diode system was demonstrated for pumping $\mathrm{Rb}$ vapor. ${ }^{17)}$ It provided $250 \mathrm{~W}$ within $20 \mathrm{pm}$ at $780 \mathrm{~nm}$.

The use of PTR Bragg mirrors for solid state lasers enabled spectral narrowing down to couple picometers with approximately the same power as for a laser with a conventional output coupler. ${ }^{18,19,20)}$ This means that spectral brightness of those lasers was increased by three orders. It is important that even for fiber lasers with large length of resonators and, therefore, small distance between longitudinal modes, it was possible to make an external resonator with a VBG output coupler and to observe single frequency operation. ${ }^{21)}$ A combination of a thick Bragg mirror and a Fabri-Perot etalon provides an opportunity to select a single transmission maximum of the etalon and enables successful operation in a single frequency regime for different rare earth ions doped solid state lasers. ${ }^{22,23)}$ The first demonstration of a monolithic etalon Fabri-Perot produced by a moiré VBG as a single maximum narrow band transmission filter in a laser resonator was recently published. ${ }^{24)}$ This approach should bring numerous opportunities for design of narrow band laser systems.

Availability of new spectral filters that could be used in laser cavities resulted in creation of different tunable narrow band lasers. It is clear that rotation of a Bragg mirror results in variation of a reflected wavelength and can be used for spectral tuning. However, this tuning requires a concurrent realignment of an additional mirror to provide feedback. It is well known that a combination of two mirrors fixed at $90^{\circ}$ is a one-dimensional retroreflector. If one of these mirrors is a Bragg mirror (Fig. 2), it is still a retroreflector. However, variation of an incident angle would change the wavelength of the reflected beam. Such a device was used as a tunable output coupler for Yb:KYW laser. ${ }^{25)}$ Emission of narrow line with $0.1 \mathrm{~nm}$ width and tunability for about $50 \mathrm{~nm}$ in vicinity of $1025 \mathrm{~nm}$ were demonstrated. Another approach for spectral tunability can be realized with the use of a transverse chirped VBG (Fig. 7) placed in a resonator as an output coupler. Sliding of this grating in direction perpendicular to the resonator axis would change a feedback wavelength. This scheme was realized for $\mathrm{Yb}$ doped fiber laser that have shown narrow emission line of $0.007 \mathrm{~nm}$ tunable within $5 \mathrm{~nm}$ range. $^{26)}$

An important opportunity is provided by the use of VBGs not for positive feedback but for rejection of spectral components that are not desirable. This approach allows decoupling the most intensive spectral components from a resonator and, therefore, to obtain lasing at week transitions of a gain medium. Laser emission at different Stark components of $\mathrm{Nd}$ in solid state lasers was successfully demonstrated. ${ }^{27)}$ It should be noted that the use of filtering in the spectral domain produces improvement of laser parameters in the time domain. Dramatic impact was produced by PTR Bragg mirrors incorporated in Nd:YLF regenerative amplifier. ${ }^{28,29)}$ Spectral filter- ing resulted in suppression of background and increasing of contrast of amplified pulse for about three orders of magnitude.

\section{Angular narrowing and steering of laser beams}

As it was described in Section 1 (Fig. 1), VBGs possess angular selectivity and therefore can serve as transverse mode selectors in laser resonators. The well-known requirement for a single-transverse-mode resonator is to provide a single Fresnel zone at an output coupler. Usually this requirement is satisfied by proper ratio between length and aperture of a resonator. However, a resonator that includes an optical element, which transmits radiation within a solid angle corresponding the first Fresnel zone, would support only a fundamental mode even for apertures significantly exceeding the size of the first Fresnel zone. Angular selectivity of VBGs was first realized for mode selection in semiconductor lasers where fast axis divergence is naturally diffraction limited while slow axis divergence is determined by interaction of large number of transverse modes. ${ }^{5,30)}$ Angular selection along the slow axis was performed by both reflecting VBGs in convergent beams and by transmitting VBGs in collimated beams. It was shown that while typical width of a semiconductor waveguide to support a single transverse mode regime should be below $5 \mu \mathrm{m}$, the external resonators with VBGs were able to keep single mode operation with waveguide widths up to $150 \mu \mathrm{m}$. Spatial brightness of laser diodes was enhanced by an order of magnitude while spectral brightness was increased by three orders of magnitude. This approach was recently applied for solid state lasers ${ }^{31)}$ where two dimensional mode selection was produced by reflecting VBGs in convergent beams and by pairs of transmitting VBGs in collimated beams. Both approaches have shown opportunities for decreasing divergence in resonators with large Fresnel numbers.

The problem of precise beam steering or receiver pointing is one of the key problems for both free space and waveguide communication systems. Large angle mechanical devices are bulky and slow, while electro-controlled devices steer within small angles. However, beam steering at small angles (below $5^{\circ}$ ) produced by conventional electro-optical, acousto-optical or liquid crystal devices can be magnified by a stack of VBGs. ${ }^{32)}$ This magnification is based on a specific profile of angular dependence of diffraction efficiency that includes a central lobe and a number of side lobes separated by zeros. This profile is similar to the spectral one depicted in Fig. 3. Deflection of a laser beam from a Bragg angle to the nearest zero causes deflection of the beam for a doubled Bragg angle. This magnification factor could exceed 100. A stack of VBGs with difference between adjacent incident angles of $0.1^{\circ}$ and difference between corresponding diffraction angles of $10^{\circ}$ was designed and realized. ${ }^{7)}$ Such approach for angular magnification in fast electro-optical switches was demonstrated and has shown dramatic decrease of their size. ${ }^{33)}$

\section{Stretching, compression and temporal shaping of laser pulses}

High power ultrashort laser pulses in picosecond and femtosecond regions are in great demand for medical and industrial applications. However, direct amplification of such pulses is restricted by different nonlinear processes. Therefore, the main method of generation of high power and high energy ultrashort pulses is chirped pulse amplification (CPA) where an ultrashort laser pulse with a corresponding wide spectrum 
is stretched by a chirped fiber Bragg grating or a pair of diffraction gratings that provide different delays for different spectral components. ${ }^{34}$ The pulse with decreased power is amplified and then compressed back by one of these devices. Both methods have restrictions resulted from small aperture of fiber Bragg gratings (and corresponding low laser damage threshold) and high sensitivity of diffraction grating pairs to misalignment (and corresponding low tolerance to vibrations and shocks).

Longitudinal chirped VBGs (Fig. 8) can produce operations with laser beam directly in the time domain. Such chirped Bragg mirror would reflect different wavelengths from different positions in direction of beam propagation. This means that reflection of different spectral components of a femtosecond laser pulse with wide spectrum would cause stretching the pulse in time and space domains. The width of a stretched pulse is determined by the delay time between extreme spectral components and it is equal to the time of double pass between corresponding points in the chirped VBG. The stretched pulse being directed to the same chirped VBG from the opposite side would be compressed back to its original state. Such gratins were fabricated and it was shown ${ }^{35,36)}$ that stretching and compression could be performed with efficiency exceeding $95 \%$. High laser damage threshold and low absorption of PTR VBGs enabled generation of high power laser pulses in CPA laser systems. ${ }^{37)}$ Efficient stretching and compression of pulses was demonstrated for all types of ultrashort pulse lasers emitting from 0.8 to $2 \mu \mathrm{m}$. Small size of VBG compressors (three orders of magnitude less volume if compared to that for a Treacy compressor) and high tolerance to shocks and vibrations enable great improvement of compactness and reliability of ultrashort CPA systems.

Additional capability to shape ultrashort pulses is provided by an opportunity to make sectional chirped gratings ${ }^{38)}$ and multipass chirped gratings with phase correctors. ${ }^{39)}$ It was found that two or more sequential chirped VBGs (Fig. 9, A and B) with adjacent reflection spectra placed with a phase shift between sections multiple of $2 \pi$ work as a single monolithic grating with such an extended reflection spectrum. This approach enables increase of total delay time and spectral width beyond current technological limits. Moreover, varying the phase shift between sections allows wide manipulations with pulse shape including double pulses, pulses with precursors, super Gaussian pulses, etc. Another method to increase stretching time is the use of chirped VBGs in a multipass regime by the use of a retroreflecting mirror (Fig. 9, C). In this

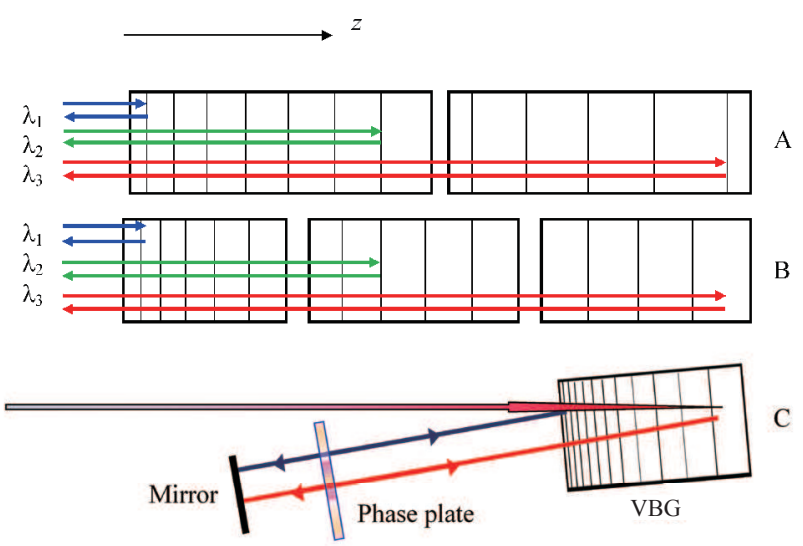

Fig. 9 Complex devices for temporal shaping ultrashort laser pulses. A - two section chirped VBG, B three section chirped VBG, C - double pass chirped VBG with phase plate. case, spectral width of the device is not changed but stretching time can be doubled, quadrupled, etc. In space between the VBG and the mirror, different spectral components of the laser pulse are dispersed in lateral direction. Therefore, placing a phase plate between the grating and the mirror provides different delays for different spectral components. This shaping in spectral domain causes reshaping in time domain.

\section{Photo-thermo-refractive laser glass and a way to monolithic solid state lasers}

A demonstration of combining a VBG with a gain element was reported long time ago. ${ }^{40)}$ A monolithic device that included a VBG, a Nd:GdVO 4 crystal and a mirror has shown a single frequency operation with tunability over $80 \mathrm{GHz}$ with no mode hoping. This idea to create a monolithic solid state laser got further development when PTR glass was co-doped with $\mathrm{Nd}^{3+}$ and it was shown that this glass provides good luminescent parameters while keeping photosensitivity. ${ }^{41)} \mathrm{CW}$ laser action in this glass was demonstrated in a plane-concave resonator with diode pumping. ${ }^{42)}$ PTR glass was successfully doped with $\mathrm{Yb}$ and Er and high efficiency reflecting VBGs were recorded in these glass plates. ${ }^{43)} \mathrm{CW}$ laser action was observed in $\mathrm{Yb}$ doped PTR glass in both distributed Bragg reflection (DBR) and distributed feedback (DFB) configurations (Fig. 10). It was found that completely monolithic solid state DFB laser emits up to $200 \mathrm{~mW}$ of single frequency radiation with spectral width about $250 \mathrm{kHz}$ that can be tuned without mode hopping for about $130 \mathrm{GHz}$. This result paves a way to extremely robust narrow band solid state lasers for a great number of applications.

\section{Laser beam combining}

It is common understanding that all types of lasers have restrictions for power scaling resulted from different nonlinear processes. Therefore, achievement of extremely high power of solid state and fiber laser systems would require different types of laser beam combining. There are two types of combining that enable increase of brightness - coherent combining, when several coherent beams are overlapped by proper phase control, and spectral combining, when several incoherent beams with different wavelengths are overlapped by proper spectral selective elements. The use of VBGs could play significant role in beam combining because of their extremely sharp spectral selectivity and their ability to provide phase
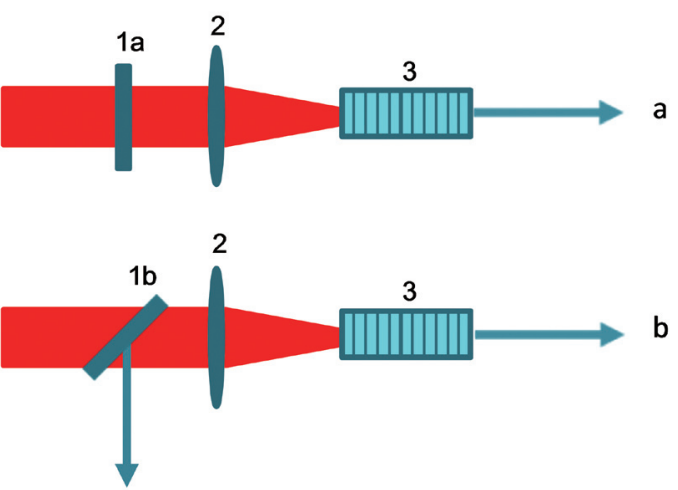

Fig. 10 Schematic of a DBR (a) and DFB (b) lasers in Yb doped PTR glass. 1a and 1b - dichroic beam splitters with HR at $1066 \mathrm{~nm}$ and HT at $976 \mathrm{~nm}$, 2- lens, 3-Yb-doped PTR glass slab with 99\% Bragg mirror. 
locking of different lasers. ${ }^{44)}$

Spectral combining is based on a specific shape of diffraction efficiency spectra of VBGs (Fig. 3) where the central maximum and side lobes are separated by zeros. This means that a beam with a proper wavelength launched to a VBG at a Bragg angle (incident beam in Fig. 2) would be diffracted. Another beam with a wavelength corresponding to one of zeros in the spectrum launched at the Bragg angle from the opposite side of the VBG (combined beam in Fig. 2) would pass the grating without diffraction and completely overlap with the first beam. For a lossless VBG, spatial brightness of a combined beam would be doubled. The sequence of such gratings designed for diffraction of specified wavelengths and transmission of all additional wavelengths provides an opportunity to combine a great number of laser beams. Figure 2 shows geometry of beam combining for a reflecting VBG. However, it is clear that a transmitting VBG can provide the same beam combining. This approach has demonstrated highest spectral brightness of combining - five beams of $\mathrm{Yb}$ doped fiber lasers with wavelength separation between adjacent channels of $0.5 \mathrm{~nm}$ were combined with efficiency exceeding $90 \%$ to a single beam with divergence close to diffraction limit and total power of $750 \mathrm{~W}^{45)}$

There are several improvements in the area of spectral beam combing. First, a set of VBGs with sharper reflection spectra enabled beam combining with similar parameters while the channel separation was narrowed down to $0.25 \mathrm{~nm}$. Second, fabrication of multiplexed VBGs (Fig. 5) enabled combining of three (by a doubled VBG) and five (by a quadrupled VBG) lasers by means of a single optical element. ${ }^{46)}$ This approach enabled dramatic decrease of a beam combiner size. Third, air cooling was demonstrated as an efficient method of increasing tolerance of VBGs to high power laser radiation. ${ }^{47)}$ It was shown that VBGs can be used in multikilowatt laser beams without degradation of diffraction efficiency and divergence of the diffracted beams.

Ability of VBGs to provide phase locking of lasers was first demonstrated with semiconductor lasers. ${ }^{48)}$ It was found that, if a spectral width of the VBG was less than the distance between adjacent longitudinal modes of internal resonators of the laser diodes, the use of a VBG for radiation exchange between two laser diodes produced phase locking and resulted in emission of coherent beams. This phase locking was stable and could be observed for a long time.

This approach was successfully used for coherent combining of fiber lasers. ${ }^{49)}$ However it is not simple to scale of this combining to multiple channels. Development of multiplexed VBGs dramatically improved the situation. This complex optical element with several VBGs having a common Bragg angle for a specified wavelength works as a beam splitter as it is shown in Fig. 6. However if this element is illuminated by two coherent properly phased beams in opposite direction, it would work as a beam combiner. This property was used for active coherent combining of five fiber lasers with total power in a combined beam of $670 \mathrm{~W}^{50)}$ Such multiplexed gratings provide even more opportunities for coherent combining. The scheme of passive coherent combining by means of multiplexed VBGs is shown in Fig. 11. ${ }^{51)}$ Radiation from all channels is diffracted by a multiplexed VBG to a common output coupler that provides feedback. Radiation reflected by the output coupler is split between channels. This scheme provides two features - radiation from an output coupler is completely mixed by a VBG before the redistribution between channels, and a combined high power beam propagates in free space not affecting fiber amplifiers. High efficiency passive

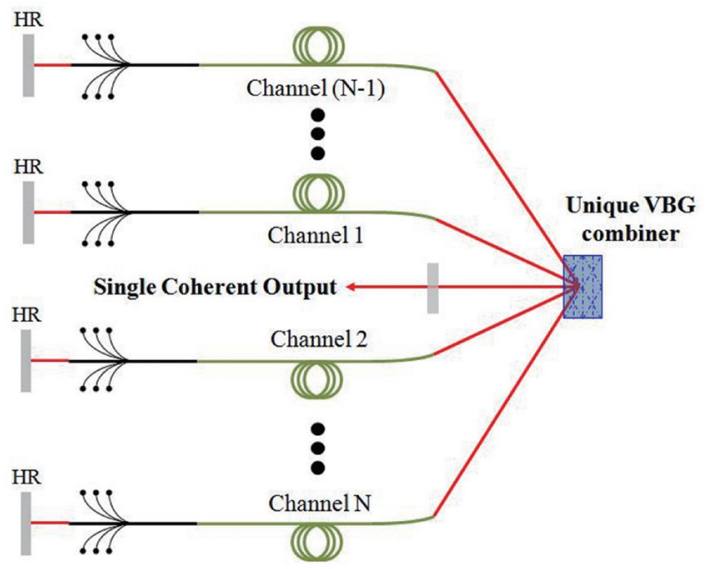

Fig. 11 Coherent locking of fiber lasers by a multiplexed reflecting VBG in a multichannel resonator with a common output coupler.

combining of two and four fiber channels was recently demonstrated. $^{\text {52) }}$

\section{Summary}

New optical elements which are volume Bragg gratings (VBGs) recorded in photo-thermo-refractive (PTR) glass enabled significant progress in laser design and applications. These elements are narrow band filters that operate in spectral, angular and temporal domains. They provide spectral narrowing, stabilization and tuning of laser radiation; narrowing of divergence and beam steering; stretching, compression and temporal shaping of laser pulses, integration of different laser components in the same material and fabrication of monolithic solid state lasers; and passive and active coherent combining along with high density spectral combining of fiber lasers.

\section{References}

1) H. Kogelnik: The Bell System Technical Journal 48 (1969) 2909.

2) L. B. Glebov: Glastech. Ber. Glass Sci. Technol. 71C (1998) 85.

3) O. M. Efimov, L. B. Glebov, L. N. Glebova, K. C. Richardson, and V. I. Smirnov: Appl. Optics 38 (1999) 619.

4) L. B. Glebov, V. I. Smirnov, C. M. Stickley, and I. V. Ciapurin: Proc. SPIE 4724 (2002) 101.

5) L. B. Glebov: Proc. SPIE 6216 (2006) 621601.

6) L. B. Glebov: Phys. Chem. Glasses: Eur. J. Glass Sci. Technol. B, 48 (2007) 123.

7) L. B. Glebov: J. of Holography and Speckle 5 (2008) 1.

8) I. V. Ciapurin, L. B. Glebov, and V. I. Smirnov: Opt. Engineering 45 (2006) 0158021.

9) I. V. Ciapurin, D. R. Drachenberg, V. I. Smirnov, G. B. Venus, and L. B. Glebov: Opt. Engineering 51 (2012) 058001.

10) V. Smirnov, J. Lumeau, S. Mokhov, B. Ya. Zeldovich, and L. B. Glebov: Opt. Lett. 35 (2010) 592.

11) I. Divliansky, D. Ott, B. Anderson, D. Drachenberg, V. Rotar, G. Venus, and L. Glebov: Proc. SPIE 8237 (2012) 823705.

12) I. Divliansky, D. Ott, B. Anderson, G. Venus, and L. Glebov: To be published in Opt. Express.

13) A. Jain, O. Andrusyak, G. Venus, V. Smirnov, and L. Glebov: Proc. SPIE 7580 (2010) $75801 \mathrm{~S}$.

14) A. Jain, D. Drachenberg, O. Andrusyak, G. Venus, V. Smirnov, and L. Glebov: Proc. SPIE 7686 (2010) 768615.

15) B. L. Volodin, S. V. Dolgy, E. D. Melnik, E. Downs, J. Shaw, and V. S. Ban: Opt. Lett. 29 (2004) 1891.

16) G. Venus, V. Smirnov, L. Glebov, and M. Kanskar: Proc. of Solid State and Diode Lasers Technical Review. Albuquerque (2004) P-14.

17) A. Podvyaznyy, G. Venus, V. Smirnov, O. Mokhun, V. Koulechov, 
D. Hostutler, and L. Glebov: Proc. SPIE 7583 (2010) 758313.

18) T. Chung, A. Rapaport, V. Smirnov, L. Glebov, M. Richardson, and M. Bass: Opt. Lett. 31 (2006) 229.

19) T. Chung, A. Rapaport, Y. Chen, V. Smirnov, M. Hemmer, L. B. Glebov, M. C. Richardson, and M. Bass: Proc. SPIE 6216 (2006) 621603.

20) M. Hemmer, Y. Joly, L. Glebov, M. Bass, and M. Richardson: Opt. Express 17 (2009) 8212.

21) A. Ryasnyanskiy, N. Vorobiev, V. Smirnov, J. Lumeau, L. Glebova, O. Mokhun, E. Rotari, Ch. Spiegelberg, A. Podvyaznyy, and L. Glebov: Proc. SPIE 8385 (2012) 838503.

22) B. Jacobsson, V. Pasiskevicius, and F. Laurell: Opt. Lett. 31 (2006) 1663 .

23) B. Jacobsson, V. Pasiskevicius, and F. Laurell: Opt. Express 14 (2006) 9284.

24) D. Ott, V. Rotar, J. Lumeau, S. Mokhov, I. Divliansky, A. Ryasnyanskiy, N. Vorobiev, V. Smirnov, Ch. Spiegelberg, and L. Glebov: Proc. SPIE 8236 (2012) 823621.

25) B. Jacobsson, J. E. Hellstrom, V. Pasiskevicius, and F. Laurell: Opt. Express 15 (2007) 1003.

26) A. Ryasnyanskiy, V. Smirnov, L. Glebova, O. Mokhun, E. Rotari, A. Glebov, and L. Glebov: In Defense, Security and Sensors Conference. Baltimore. MD, 2013. Proc. SPIE, 2013, to be published.

27) N. S. Vorobiev, L. B. Glebov, V. I. Smirnov, and I. S. Ciapurin: Quant. Electronics 39 (2009) 43.

28) A. V. Okishev, C. Dorrer, V. I. Smirnov, L. B. Glebov, and J. D. Zuegel: Opt. Express 15 (2007) 8197.

29) C. Dorrer, A. V. Okishev, I. A. Begishev, J. D. Zuegel, V. I. Smirnov, L. B. Glebov: Opt. Lett. 32 (2007) 2378.

30) G. B. Venus, A. Sevian, V. I. Smirnov, and L. B. Glebov: Proc. SPIE 5711 (2005) 166.

31) B. Anderson, G. Venus, D. Ott, I. Divliansky, and L. Glebov: Conference "Solid State and Diode Laser Technology Review", Broomfield, CO. 2012/06/11.

32) D. P. Resler, D. S. Hobbs, R. C. Sharp, L. J. Friedman, and T. A. Dorschner: Opt. Lett. 21 (1996) 689.

33) A. L. Glebov, A. Sugama, V. I. Smirnov, S. Aoki, V. Rotar, M. G. Lee, and L. B. Glebov: IEEE Phot. Techn. Lett. 19 (2007) 701.

34) E. B. Treacy: IEEE J. Quant. Electron. 5 (1969) 454.

35) V. Smirnov, E. Flecher, L. Glebov, K. Liao, and A. Galvanauskas: In Proc. of Solid State and Diode Lasers Technical Review. Los
Angeles 2005, SS2-1.

36) K. Liao, M. Cheng, E. Flecher, V. Smirnov, L. Glebov, and A. Galvanauskas: Opt. Express 15 (2007) 4876.

37) M. Rever, S. Huang, V. Smirnov, E. Rotari, I. Cohanoshi, S. Mokhov, L. Glebov, and A. Galvanauskas: Proc. SPIE $\mathbf{7 5 8 0}$ (2010) $75801 Y$.

38) O. Andrusyak, L. Canioni, I. Cohanoshi, M. Delaigue, E. Rotari, V. Smirnov, and L. Glebov: Proc. SPIE 7578 (2010) 75781A.

39) D. Moses, O. Andrusyak, M. SeGall, J. Lumeau, V. Smirnov, V. Rotar, and L. Glebov: Advanced High Power Laser Review. 8th Annual Ultrashort Pulse Laser Workshop. Broomfield, CO. June $17,2010$.

40) I. Haggstrom, B. Jacobsson, and F. Laurell: Opt. Express 15 (2007) 11589.

41) L. Glebova, J. Lumeau, and L. B. Glebov: Opt. Materials 33 (2011) 1970.

42) Y. Sato, T. Taira, V. Smirnov, L. Glebova, and L. Glebov: Opt. Lett. 36 (2011) 2257.

43) L. Glebov, A. Ryasnyanskiy, V. Smirnov, L. Glebova, O. Mokhun, A. Glebov, J. Lumeau, S. Vasu, and M. J. Soileau: Optics and Photonics International Congress. Laser Ignition Conference. Paper LIC3-4. Yokohama, Japan. 2013/04/24

44) L. B. Glebov: Proc. SPIE 6216 (2006) 621601.

45) O. Andrusyak, V. Smirnov, G. Venus, V. Rotar, and L. Glebov: IEEE J. of Selected Topics in Quant. Electronics 15 (2009) 344.

46) I. Divliansky, D. Ott, B. Anderson, D. Drachenberg, V. Rotar, G. Venus, and L. Glebov: Proc. SPIE 8237 (2012) 823705.

47) B. Anderson, S. Kaim, G. B. Venus, J. Lumeau, V. Smirnov, B. Zeldovich, and L. Glebov: Proc. SPIE 8601 (2013) 86013D.

$48)$ G. B. Venus, A. Sevian, V. I. Smirnov, and L. B. Glebov: Opt. Lett. 31 (2006) 1453.

49) A. Jain, O. Andrusyak, G. Venus, V. Smirnov, and L. Glebov: Proc. SPIE 7580 (2010) 75801S.

50) C. A. Lu, A. Flores, E. J. Bochove, W. P. Roach, V. Smirnov, and L. B. Glebov: Proc. SPIE 8601 (2013) 86011A.

51) A. Jain, D. Drachenberg, O. Andrusyak, G. Venus, V. Smirnov, and L. Glebov: Proc. SPIE 7686 (2010) 768615.

52) A. Jain, C. A. Lu, G. Venus, V. Smirnov, E. Bochove, and L. Glebov: Photonics West Conference, San Francisco 2013. Paper 8601-46. 